\title{
Multifractal analysis of oil slicks on SAR images
}

\author{
Roberto Coscione, Gerardo Di Martino, Antonio Iodice, Daniele Riccio, Giuseppe Ruello \\ Università di Napoli “Federico II”, DIBET, Via Claudio 21, 80125, Napoli, Italy
}

\begin{abstract}
In this paper we present a technique for the analysis of low intensity patches on SAR oceanic amplitude images. The proposed technique, which is based on multifractal analysis of the edges of dark areas (here called regions of interest, ROIs), can be used to identify oil slicks generated by moving ships. The core idea is that different physical-chemical interactions of oil slicks and look-alikes with the sea surface imply different multifractal features for the edges of the ROIs on the acquired images. Accordingly, we propose to perform a multifractal analysis on ROIs' edges, which consists in the estimation of their multifractal spectrum and in the evaluation of the "dispersion area" of this spectrum. The proposed procedure is tested on simulated SAR images and methods and results are extensively discussed. First results seem to indicate that the observation of multifractal spectra is useful in order to distinguish between oil slicks generated by moving ships from other kinds of slicks, even when these phenomena have the same degree of irregularity and an estimation of the classical fractal dimension is not suitable for discrimination purposes.
\end{abstract}

Keywords: SAR, oil slicks, multifractal analysis

\section{INTRODUCTION}

Synthetic Aperture Radar (SAR) systems offer incredible capability for earth observation and specifically for sea monitoring, which could, at least in principle, fill the lack of in-situ pollution surveys of seas and coasts and offer the ability to monitor oil slick occurrences. Oil slicks on the ocean surface can be observed in SAR images as dark spots. Nevertheless, the use of SAR data is still limited because dark areas in the images can be due to several phenomena, as lack of wind, natural oil, plankton, and so on. So far, this ambiguity limited the development of automatic oil detection procedure.

The interpretation of SAR images can be strongly improved if reliable direct models, accounting for the dominant physical phenomena (wind history, slick nature, sensor characteristics, and so on) are available. In this framework, the fractal geometry is widely recognized as the most appropriate instrument for the description of natural shapes [1]. In this paper we deal with a generalization of fractal geometry, the so-called multifractals, which present different fractal characteristics according to the observation scale [1]. Hence, while fractal processes can be described through their fractal dimension, the multifractals present a continuous distribution of fractal dimensions and are described more effectively through their multifractal spectrum. The introduction of multifractals is convenient when the analysis of oil slicks on the sea surface is in order: in fact, different physical-chemical interactions of oil slicks and look-alikes with the sea surface imply different features for the edges of the dark spots (in the following called regions of interest, ROIs) on the acquired images. In particular, the shape of the oil slicks due to illegal tank-cleaning from moving ships (at least those whose generation is close to the time of observation) present features which are determined by concurrent turbulent phenomena acting at different magnitude scales, i.e., ship movement, that determines typical elongated shape of related oil slick, sea turbulence due to ship's engines, as well as natural sea turbulence, thus implying a multifractal feature of ROIs' edges. Conversely, look-alikes due to lack of wind present a shape that is related to the wind turbulent behavior, implying a monofractal feature of ROIs' edges. Moreover, oil slicks not generated by moving ships, or slicks which stay on the sea surface for a long time, present a shape dictated essentially by sea turbulence only, implying a monofractal feature of ROIs' edges, as well.

In this paper we propose the use of multifractal analysis of oil slicks edges, which is based on the estimation of their multifractal spectra and on the definition of their "dispersion areas", in order to identify the oil slicks due to illegal tankcleaning. The proposed procedure can be tested on simulated SAR images, obtained by a two-step simulation scheme. First, a combination of deterministic and stochastic models (the latter based on fractional Brownian motion, fBm) are used to describe the contour of oil slicks and look-alikes [2]. Then these contours are given as input to a SAR simulator [3] able to provide simulated SAR images relevant to sea surfaces covered by slicks of different nature. Such a simulator

SAR Image Analysis, Modeling, and Techniques XI,

edited by Claudia Notarnicola, Simonetta Paloscia, Nazzareno Pierdicca, Proc. of SPIE Vol. $8179,81790 \mathrm{H}$

(C) 2011 SPIE · CCC code: 0277-786X/11/\$18 - doi: 10.1117/12.898153

Proc. of SPIE Vol. $817981790 \mathrm{H}-1$ 
relies on efficient models of the ocean description and of the electromagnetic-ocean wave interaction. The ocean surface is described by means of a two scale model, including long (periods of about 100 meters), and short (periods of $\mathrm{cm}$ and $\mathrm{mm}$ ) waves. In addition, intermediate scale waves are accounted for as an effect on the signal. A model based on the Marangoni theory accounts for the oil slick effect as well [3]. Finally, on these simulated images the above mentioned multifractal analysis can be applied after a preliminary elaboration step, consisting in the extraction of the ROIs' edges. Actually, this step can be performed using different techniques (e.g. thresholding, CFAR approaches [4], and morphological operators [2]), thus obtaining the contour of the slick with an accuracy which is also related to sea and wind conditions. Anyway, for the scope of this paper we are not interested in this preliminary elaboration step and, hence, we assume that a perfect reconstruction of the ROIs' edges has been obtained and apply the multifractal analysis on these "optimum" contours. An extensive discussion on the methods used for elaboration and on the obtained results is also presented. In particular, the box-counting method is adopted for the evaluation of the multifractal parameters and a study on the choice of boxes dimension range is also performed, emphasizing the critical role of this processing step.

The paper is organized as follows. In Section 2 the geometrical fractal and multifractal models are presented. In Section 3 the rationale of SAR simulation is recalled and the simulated data are described. In Section 4 the obtained results are shown: they indicate that the observation of multifractal spectra is useful in order to distinguish between oil slicks generated by moving ships from other kinds of slicks, even when these phenomena have the same degree of irregularity and an estimation of the classical fractal dimension is not suitable for discrimination purposes. Finally, in Section 5 significant conclusions are drawn.

\section{GEOMETRICAL MODELS}

Fractal geometry is widely recognized as the most appropriate instrument for the modeling and the analysis of natural shapes. Fractals allow the description of complex natural objects, both in the one-dimensional (e.g., the path of a river, the contour of islands or lakes) and multi-dimensional (such as natural surfaces and snow) cases, using a minimum number of independent parameters. In the following we describe first the fractal models used in this work to obtain the shapes of interest for the simulated slicks. Then the key concepts of multifractal analysis are detailed, with a particular focus on the method of moments used in this work for their description and analysis.

\subsection{Fractal models}

It is very useful for testing oil detection techniques, to generate slicks with fractal shapes on simulated SAR data. In order to do it, the most appropriate function is the fractional Brownian motion ( $\mathrm{fBm}$ ), which is a process described in terms of the pdf of its increments [1]. In this paper we synthesized realizations of fBm processes by means of the twodimensional Weierstrass-Mandelbrot (WM) function, which is a superposition of sinusoidal tones, spaced by means of an irrational parameter $v$.

$$
z(x, y)=A \sum_{p=0}^{M-1} C_{p} v^{-H \cdot p} \sin \left[k_{0} v^{p}\left(x \cos \Psi_{p}+y \sin \Psi_{p}\right)+\Phi_{p}\right],
$$

where $A$ is the vertical overall height scaling factor, $C_{p}, \Phi_{p}, \Psi_{p}$, are random amplitude, phase and direction coefficients, $H$ is the Hurst coefficient $(0<H<1)$, related to the fractal dimension $D_{W M}$ by means of the relation $D_{W M}=3-H, k_{0}$ the fundamental wavenumber.

A cut of the WM function at fixed $z$ levels provides a fractal curve (with fractal dimension equal to $D_{W M}$ decreased by one) which defines the region covered by the oil. As an example, in Fig. 1 we depict a WM function with fractal dimension $D_{W M}=2.3$ (a), one slick obtained from a cut of this WM (b) and the contour extracted from the slick (c). The image was synthesized on a $3.99 \times 19.9 \mathrm{~m}^{2}$ spaced grid, in accordance with the ERS-1 acquisition geometry. The image pixel of Fig.1 has a 19.9m x 19.9m (azimuth $\times$ range) dimension, in order to show the ground aspect of the slick. Therefore, such an image can be used as an input for the simulation of ERS-1 raw signals and images.

The fractal dimension of the slick can be computed using box counting techniques, as detailed in [1]-[2]. This technique is based on the fact that if we cover a fractal set with non-overlapping boxes containing at least one point belonging to the set we obtain the following relation: 


$$
N_{b}(\delta) \propto \delta^{-D_{B C}}
$$

where $N_{b}(d)$ is the number of non-empty boxes of dimension $\delta$ and $D_{B C}$ is the box counting dimension, which is an estimate of the fractal dimension of the set and can be evaluated as

$$
D_{B C}=\lim _{\delta \rightarrow 0} \frac{\log N_{b}(\delta)}{\log \delta} .
$$

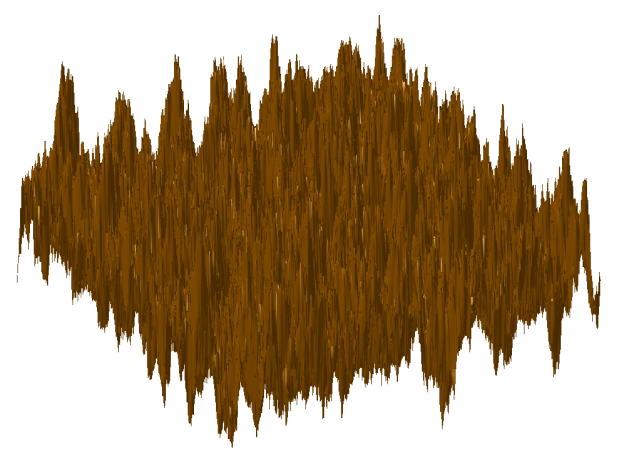

(a)

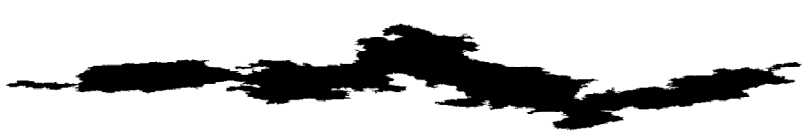

(b)

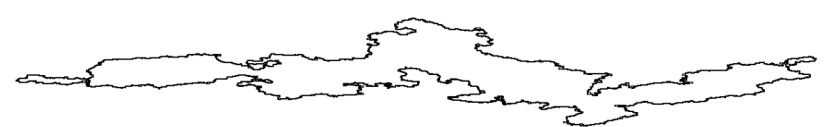

(c)

Figure 1. Simulation of fractal shapes: (a) WM surface with Hurst parameter equal to 0.7 used for to extract the shapes used in tests; (b) two-dimensional shape obtained intersecting the WM surface with a fixed z-plane; (c) fractal contour used in the tests.

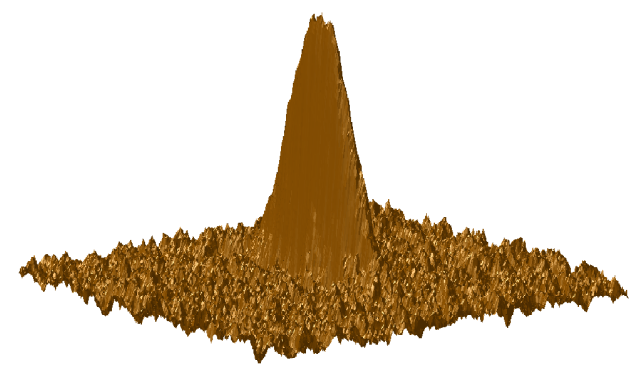

(a)

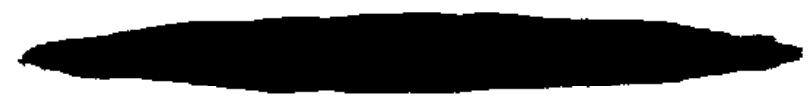

(b)

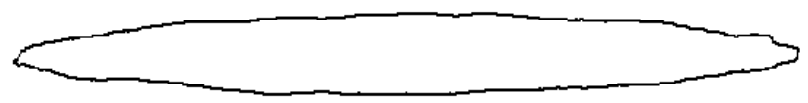

(c)

Figure 2. Models used to simulate multi-fractal shape: (a) WM surface with Hurst parameter equal to 0.7 superimposed to a Gaussian function; (b) two-dimensional shape obtained intersecting the previous surface with a fixed $z$-plane; (c) fractal contour used in the tests. 
The dark spot of Fig. 1 can be effectively used to model the behavior of natural spills and of man-made slicks when a long time from their generation has elapsed. Conversely, in Fig. 2 a dark spot which can be used to model slicks due to illegal tank-cleaning is shown. It was obtained superimposing a Gaussian function presenting standard deviations in two orthogonal directions very different from each other (one order of magnitude) to the above introduced WM: the obtained surface is shown in Fig. 2 (a), along with the dark spot obtained from a cut (b) and the contour extracted from this slick (c). However, in this case the extracted curve does not hold the same fractal behavior at all the involved spatial scales. Thus a different mathematical instrument is necessary in order to adequately model the problem at hand. In the following sub-section the rationale of multifractal processes description and analysis is summarized.

\subsection{Multifractal models}

Multifractal sets are a generalization of fractal sets to the case of non-unique fractal dimension at all scales [1]. Hence, an adequate description of their behavior requires the definition of a continuous distribution of fractal dimensions. Different methods are available in the literature for the description and analysis of multifractal sets. In this paper we use the method of moments, which is detailed in the following.

We define the moment of order $q, \chi(q, \delta)$, as

$$
\chi(q, \delta)=\sum_{i=1}^{N_{b}(\delta)} \mu_{i}(\delta)^{q},
$$

where $N_{b}(\delta)$ is the number of $\delta$-boxes for which $\mu_{i}(\delta)>0$ (i.e., non-empty boxes); $\mu_{i}(\delta)$ is the probability that a point belonging to the set falls into the $i$-th $\delta$-box and $q \in \mathfrak{R}$ is the order of the moment.

It can be show that in many cases of interest the function in (4) presents the following scale behavior:

$$
\chi(q, \delta) \propto \delta^{\tau(q)},
$$

where $\tau(q)$ is the $q$-th order mass exponent, which, according to (5), can be computed as

$$
\tau(q)=\lim _{\delta \rightarrow 0} \frac{\log [\chi(q, \delta)]}{\log (\delta)} .
$$

Note that (5) is the multifractal analogous of (2). Thus, a generalized fractal dimension can be defined as

$$
D(q)=\frac{\tau(q)}{q-1} .
$$

In case of monofractal sets $D(q)$ is constant with $q$, while for multifractals it is a monotonic decreasing function of $q$, presenting an horizontal asymptote for $q \rightarrow \infty$. It is easy to realize that substituting $q=0$ into (7) we obtain the canonical box counting dimension of (3).

Now we can define the local Holder exponent, $\alpha(q)$ as

$$
\alpha(q)=\frac{\mathrm{d}}{\mathrm{d} q} \tau(q) .
$$

The multifractal spectrum, $f(\alpha)$, can be introduced according to the following expression:

$$
f(\alpha)=q \alpha(q)-\tau(q) .
$$

This means that a multifractal set can be divided into subsets of index $\alpha$, whose fractal dimension is equal to $f(\alpha)$. For a multifractal set the graph of $f(\alpha)$ is convex with maximum for $q=0$, where it assumes a value equal to $D_{B C}$. Due to the fact that for monofractal sets $\alpha$ assumes a unique value, for these sets the multifractal spectrum degenerates into a point. This observation is the starting point for the development of our technique: in fact, we propose to use the multifractal spectrum of ROIs' edges (and in particular a synthetic index that we called the dispersion area) in order to improve the 
accuracy in the identification of oil slicks due to illegal tank-cleaning, which, as mentioned above, present in general a multifractal behavior.

\section{SAR SIMULATION OF OCEAN SCENES}

In this section we recall the most important concepts for the SAR raw signal simulation of oil slicks on the ocean surface and we show the simulations relative to oil slicks presenting the shapes described in the previous section.

Let $t$ be the independent time variable, $x$ and $r$ the independent space variables, standing respectively for azimuth and range. By using prime coordinates for the SAR raw signal, this can be expressed as [3]

$$
s\left(x^{\prime}, r^{\prime}\right)=\iint d x d r \gamma\left(x, r, x^{\prime}=v_{s} t\right) g\left(x^{\prime}-x, r^{\prime}-r ; r\right),
$$

wherein $\chi(\cdot)$ is the (time-varying) reflectivity pattern of the ocean, $v_{s}$ the sensor velocity, and $g(\cdot)$ the unit impulse response of the SAR system.

The evaluation of the reflectivity function requires a description of the ocean scene and a model of the interaction between electromagnetic and ocean waves. Here the ocean surface is described as a superposition of a swell and a roughness of the order of the incident wavelength. In addition, the area covered by the oil can be defined according to the shapes presented in Section 2. Moreover, the time variant nature of the ocean, in our simulator, is included via the Harger DS model. According to this model, the time dependence can appropriately "migrate" from the reflectivity function to the impulsive response function provided that a dispersion relation for the long wave spectrum is available [3]. As for the electromagnetic model, in ocean environment, the SAR signal intensity turns out to be proportional to the ocean spectrum at spatial frequencies given by the Bragg theory. The oil influence is accounted for via the Marangoni theory [3], as a damping of the ocean spectrum. The intensity of the damping depends on the oil properties and it is presents a resonant behaviour. Therefore, the best sensor for oil detection depends also by the oil resonant frequency. As an example, the Oleyl Alchool, which is here considered as case study, presents a resonance around spatial frequency that corresponds to the C-band. The SAR unit impulse response is evaluated once the sensor parameters are defined.

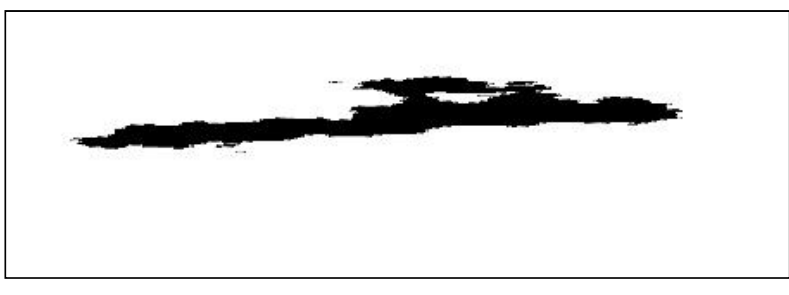

(a)

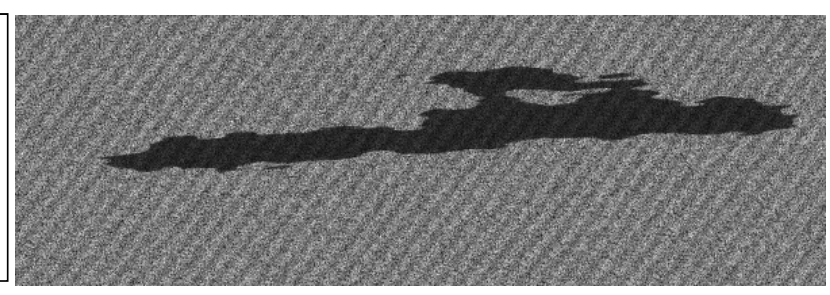

(b)

Figure 3. Fractal mask given as input to the simulator (a) and the corresponding simulated image (b).

The C-band ERS-1 SAR image relevant to the oil slick whose shape is shown in Fig. 3 (a) is obtained from the simulated raw signal (10) via standard processing and presented in Fig. 3 (b). The image is averaged with a $1 \times 4$ multi-look, so that its resolution in azimuth - ground range corresponds to an approximately square pixel of $19.9 \times 19.9 \mathrm{~m}^{2}$.

The introduced simulator can also be exploited to account for a typical ambiguity problem, related with the lack of wind. In fact, it is possible to generate arbitrary shaped regions where the wind intensity is reduced with respect to the background.

As mentioned in the introduction, in this paper we are interested in the investigation of the potentialities of multifractal concepts applied to the analysis of the ROIs' edges. Hence, in the following we use the contours extracted directly from the masks, without performing the extraction on the simulated images. In this way we can disregard the dependence from the considered extraction technique, obtaining an upper-bound for the performances of our analysis techniques. Anyway, 
a discussion on the retrieving of ROIs' edges from SAR images and the effects on the estimation of their fractal dimension can be found in [2].

\section{RESULTS}

In this section the multifractal analysis, whose basic concepts were introduced in Section 2, is applied on ROIs' edges in order to test the possibility of identifying the dark spots due to illegal tank-cleaning (when this event occurred close to the time of observation).

The first parameter we could think to use as a slick automatic classifier is the fractal dimension, which can be estimated using the box counting dimension in (3). Obviously the number of box dimensions used for the implementation of (3) must be limited to a finite range, whose maximum and minimum can be set according to the maximum and minimum dimensions of the profile under elaboration: anyway, an analogous problem will be faced in the following for the multifractal algorithms implementation. In Fig. 4 we show the log-log plots which are used to perform a linear regression in order to estimate the box counting dimension: in particular, Fig. 4 (a) is relevant to a monofractal contour, while in Fig. 4 (b) the graph relevant to a multifractal is presented. In the former case the estimated value of the fractal dimension is equal to $D_{b c}=1.3$, while in the latter case we obtain $D_{b c}=1.22$, whereas the fractal dimension of the WM surface used in both cases is equal to 1.3. Hence, for the monofractal the obtained value is very accurate; however, the difference in the estimated box counting dimension between the monofractal and the multifractal does not allow a stable and effective classifier for the observed slicks. This can be noted also observing the similar shapes of the graphs in Fig.4, whose behaviors appear to be linear in both cases. The difference between the two estimated dimensions is not stable when computed over different multifractal contours realizations obtained starting from a fractal surfaces presenting the same fractal dimension. Moreover, the box counting dimension computed on multifractal shapes always falls in the same range of that of the monofractal ones, not allowing automatic discrimination. We conclude that in actual situations the standard box counting estimation is not an appropriate technique for the classification of oil slicks.

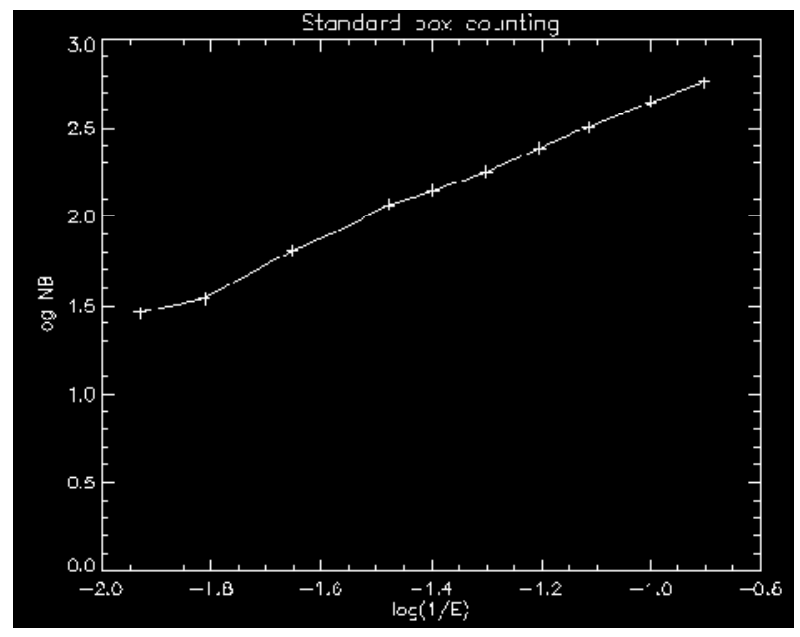

(a)

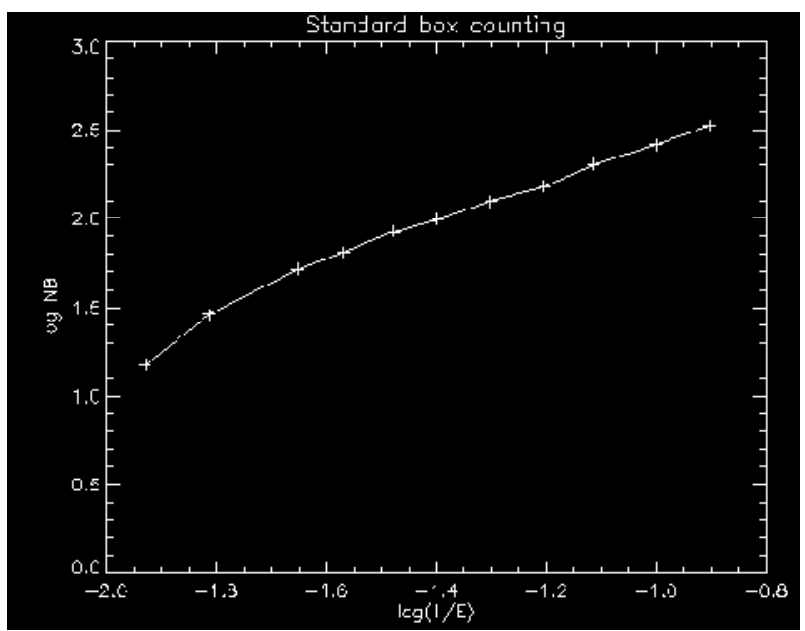

(b)

Figure 4. Log-log plots used for the standard box counting evaluation: (a) monofractal contour; (b) multifractal contour.

In Fig. 5 the multifractal spectra relevant to the same contours used in Fig. 4 are shown. These were computed setting in (4):

$$
\mu_{i}(\delta)=\frac{m_{i}(\delta)}{N_{c}}
$$


where $m_{i}$ is the number of contour pixels belonging to the $i$-th $\delta$-box and $N_{c}$ is the total number of contour pixels.

In particular, in Fig. 5 (a) the monofractal contour is considered, while in Fig. 5 (b) the multifractal contour is analyzed. It is evident from the graphs, that in the monofractal case the multifractal spectrum is much more concentrated around a point: in fact, as mentioned in Section 2, it should ideally be a single point whose value is equal to the box counting dimension. In the multifractal case the spectrum is much more dispersed. Hence, we can define a measure of this dispersion, a dispersion area, $A_{d}$ :

$$
A_{d}=\sigma_{f} \cdot \sigma_{\alpha},
$$

wherein $\sigma_{f}$ and $\sigma_{a}$ are the standard deviations of $f$ and $\alpha$, respectively. For the considered case study the values of $A_{d}$ for the monofractal and multifractal case are equal to $1.65 \times 10^{-3}$ and $41.70 \times 10^{-3}$, respectively. Therefore, for the mutifractal we obtain a dispersion area which is more than one order of magnitude greater with respect to the monofractal.

Some comments on the choice of the multifractal analysis parameters are now in order. The first parameters whose choice is fundamental in order to obtain correct estimates of the multifractal spectra are the number and dimensions of the used boxes. The upper limit for this dimension must be about one order of magnitude smaller than the maximum linear dimension of the considered ROI, while the lower limit should be one order of magnitude greater with respect to the minimum linear dimension of the considered ROI (which is usually the pixel, at least when no interpolation or resampling has been performed on the ROIs' edges). Whenever the choice of the range of box dimensions is done according to these simple rules, the number of boxes used for our analysis is not a key parameter, i.e. assuming that at least boxes presenting five different dimensions are used, an increase in their number does not imply a significant change in the obtained results. In the case study proposed in Fig. 4 and 5 the optimum range of dimensions and number of boxes were set.

Another parameter of interest is the order of the moments, $q$, used as an estimate of (9). In the proposed case study was set $q \in[0,10]$ and only integer values were considered. When the upper limit for $q$ is set to an integer lower than 5 the dispersion area of the multifractal spectrum relevant to the multifractal ROIs' edge significantly decreases and the proposed technique results in an increased probability of missing the oil slicks of interest. A significant dispersion of the multifractal spectra is obtained for $q>5$.

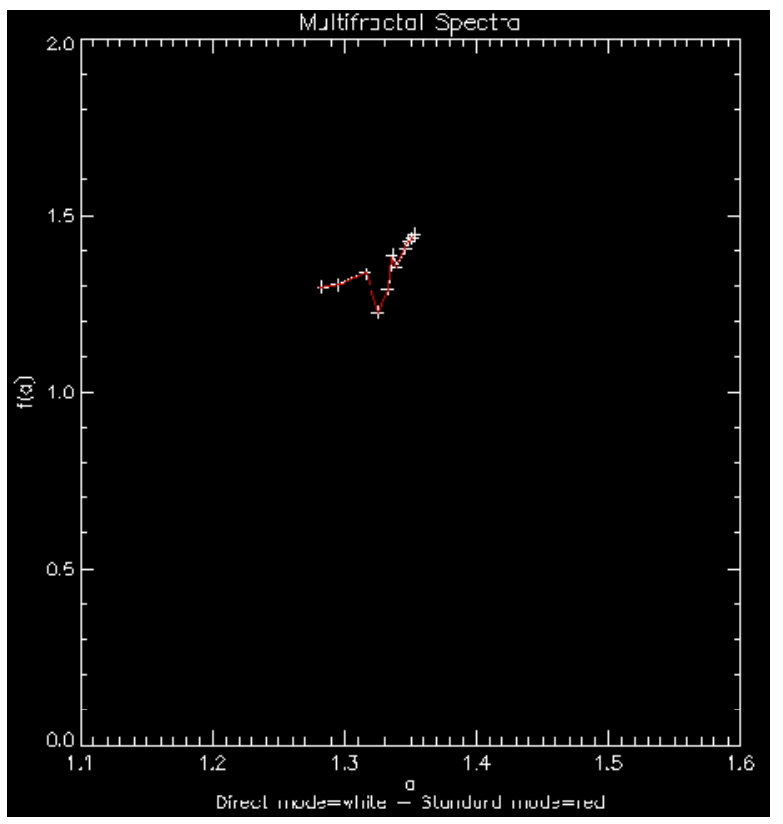

(a)

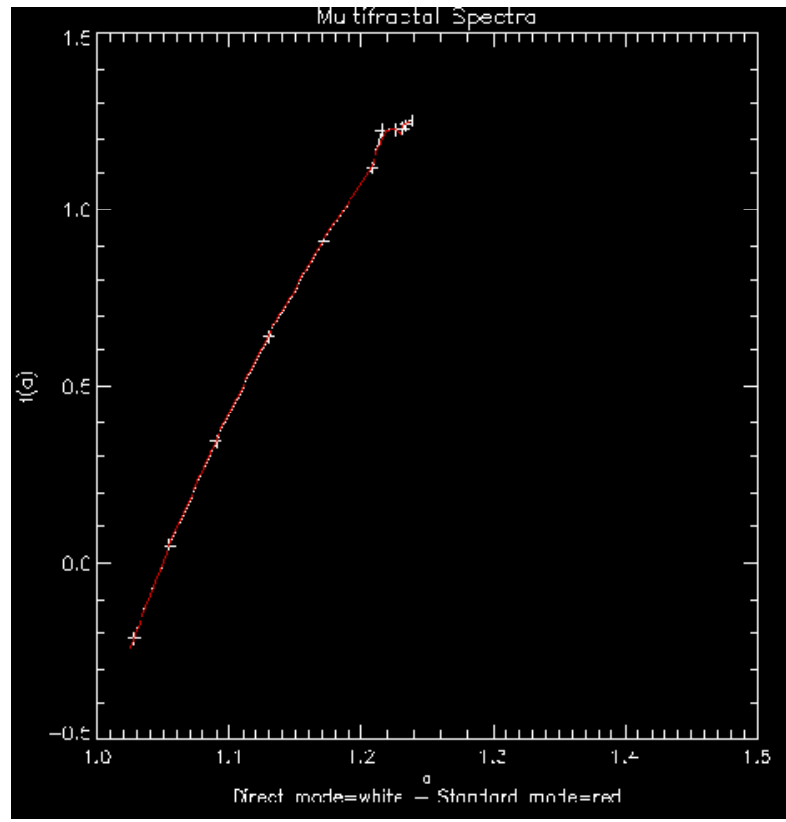

(b)

Figure 5. Multifractal spectra: (a) monofractal contour; (b) multifractal contour. 
In all the tests we made using the mentioned optimum choices for the multifractal analysis parameters, we obtained a dispersion area for the monofractal and multifractal slicks similar to that obtained for the presented case study. In conclusion, the proposed technique can be used as an effective automatic classifier for the oil slicks due to illegal tankcleaning.

\section{CONCLUSIONS}

In this paper we described the rationale of multifractal analysis applied to the study of dark spots contour on SAR images relevant to oceanic scenes. We presented a method for the generation of monofractal and multifractal shapes based on the synthesis of WM surfaces. A SAR raw signal simulator able to provide images of the sea covered by oil slicks of arbitrary shapes was also introduced.

The potentiality of the multifractal spectrum as a slick classifier were highlighted and tested on simulated slicks. The dispersion area of the multifractal spectrum as a compact segmentation index was defined. Finally, the role of the multifractal analysis parameters and the criteria for their optimum choice were investigated. The obtained results show that the analysis of the multifractal spectrum and of its dispersion area can be used as effective classifiers for the identification of slicks due to illegal tank-cleaning on SAR images.

\section{ACKNOWLEDGMENTS}

This work has been supported in part by Agenzia Spaziale Italiana within COSMO/SkyMed AO, project ID 1180.

\section{REFERENCES}

[1] Falconer, K. [Fractal Geometry], Wiley, Chichester, (1989).

[2] Danisi, A., Di Martino, G., Iodice, A., Riccio, D., Ruello, G., Tello, M., Mallorqui, J.J., Lopez-Martinez, C. , "SAR Simulation of Ocean Scenes Covered by Oil Slicks with Arbitrary Shapes", Proc. IGARSS 2007, 13141317 (2007).

[3] Franceschetti, G., Iodice, A., Riccio, D., Ruello, G., Siviero, R., "SAR Raw Signal Simulation of Oil Slicks in Ocean Environment", IEEE Trans. Geosci. Remote Sens., 40(9), 1935-1949, (2002).

[4] di Bisceglie, M., Galdi, C., "CFAR detection of extended objects in high-resolution SAR images", IEEE Trans. Geosci. Remote Sens., 43(4), 833-843, (2005). 